\title{
Novel Dynamics are Represented as a Mixture of Multiple Coordinate Systems
}

Raz Leib, David W. Franklin

3

4 Abbreviated title: Mixed Coordinate System Representation of Dynamics

6 Affiliation:

7 Neuromuscular Diagnostics, Dept. of Sport and Health Sciences, Technical University of

8 Munich, 60/62 Georg-Brauchle-Ring 80992 Munich, Germany

10 Corresponding Author: David W. Franklin (david.franklin@ tum.de)

11

12 Conflict of Interest: The authors declare no competing financial interests.

13 Acknowledgments: We thank Christian Stockinger for his contributions to an early version of this 14 experiment. 


\section{Abstract}

16 Skillful and accurate object manipulation requires the object's dynamics to be represented within

17 a motor memory. Despite extensive research, how the motor system represents novel dynamics is

18 still an open question. This representation is normally examined by testing the spatial

19 generalization of the learned forces. However, previous studies have not been able to provide a

20 consistent view of this representation in the motor system, with each study providing evidence

21 favoring an extrinsic coordinate system, an intrinsic coordinate system, an object-based

22 representation, or mixtures of these coordinate systems, but no study could definitively exclude

23 other possibilities. Most previous studies used a skew viscous force field, but these force fields

24 limit the use of channel trials and can therefore prevent us from distinguishing between different

25 coordinate systems. Here we propose a novel method to overcome these disadvantages, by

26 developing a new force field in which a curl force field is scaled by a trigonometric function of

27 movement direction allowing precise assessment of the learned generalization. In a series of four

28 experiments we show that the representation of ambiguous external dynamics is formed by a

29 mixture of extrinsic, intrinsic and object-based coordinate systems with a slightly higher weighting

30 for the object-based representation compared with Cartesian or joint representations. These results

31 highlight the existence of multiple representations in the motor system but more importantly it

32 shows that for certain dynamics the motor system prefers representation using a mixture of

33 representations over single frame based representation. 


\section{Introduction}

35 During object manipulation, such as swinging a jump rope, we experience external forces. To 36 manipulate the object, we need to estimate the mapping between the motion and the resulting

37 forces, and then send appropriate commands to the muscles that compensate for the arm and object 38 dynamics. While this seems trivial, it is still unknown how we represent the dynamic mapping of 39 such tasks, as there are at least three different reference frames that can solve this problem. First, 40 we could use a Cartesian coordinate frame in which the forces are represented as vectors in a 41 coordinate system located externally to the body. Second, we could represent the forces using the 42 shoulder, elbow and wrist joint torques. Third, we could calculate the forces using the orientation

43 vector connecting between the shoulder and the object or hand in space (Berniker et al., 2014).

44 One way to distinguish between these representations is by examining how dynamics are 45 generalized in space. In dynamic generalization, participants learn to move under force 46 perturbations (Shadmehr and Mussa-Ivaldi, 1994) followed by testing how the learned dynamics

47 are extrapolated to new locations in space. Although many studies have used dynamic 48 generalization to address the topic of representation, the results do not provide a consistent view 49 of the coordinate frame used by the motor system. Some studies support a Cartesian based 50 representation (Criscimagna-Hemminger et al., 2003), while others support a joint-based 51 (Shadmehr and Mussa-Ivaldi, 1994) or object-based representation (Yeo et al., 2015; Franklin et 52 al., 2016). In addition, some studies suggested that the system uses a mixture of the aforementioned 53 coordinate frames (Parmar et al., 2011; Berniker et al., 2014). However, even in these studies a 54 range of possible coordinate frames could explain the data. For example, although Berniker et al. 55 (2014) suggested that the mixture of coordinate frames described adaptation, they were unable to 56 distinguish between five different models, including both single coordinate frame models, and 57 ones with and without spatial decay. The lack of consistency between these results raises the 58 question as to the true nature of this internal representation.

59 We suggest that one reason for this inconsistency in results is that most studies used a velocity 60 dependent force field implemented using a skew matrix. That is, for most movement directions, 61 the forces applied on the hand consist of both forces perpendicular to movement direction and 62 (assistive or resistive) forces in the same direction as the movement. In such cases, two major 63 problems complicate detection of generalized forces. First, we are not able to accurately measure 
64 the generalized forces. Usually, to measure these forces we apply a force channel (Scheidt et al., 65 2001), a virtual force field constraining the hand to move in a straight path to the target. The applied

66 forces on the virtual channel are the forces that the motor system generates to contradict the

67 expected external forces. While this method has great advantages, it cannot detect the forces in the

68 skew force field that are not perpendicular to movement direction. Second, a skew force field will

69 produce assistive or resistive forces in the direction of movement, which can alter the movement

70 kinematics when moving in a force channel (Berniker et al., 2014). This could cause the participant

71 to miss the target, but more importantly could change the spatial or temporal nature of the

72 generalized forces.

73 To overcome these disadvantages, we used a velocity dependent curl force field, which generates

74 perpendicular forces on the hand, and by scaling differently the forces according to movement

75 direction, forces generated along one motion direction differ in amplitude and sign compared with

76 other directions. Critically, this variation in the forces means that we can examine the directional

77 change of the generalized forces compared with the learned force to distinguish between different

78 representations. Here we show that the representation of ambiguous novel dynamics is formed by

79 a mixture of coordinate frames with a slightly higher weighting for the object-based representation. 


\section{Methods}

Participants

82 Thirty-two right-handed participants (14 females; aged between 22-35) participated in one of four 83 experiments after signing a consent form and completing a handedness test (Oldfield, 1971). The

84 experimental protocol was approved by the Ethics Committee of the Medical Faculty of the

85 Technical University of Munich. Each participant only performed one of the four experiments.

87 We created a haptic augmented virtual reality environment using a vBOT robotic device (Howard et al., 2009). The robotic device was used to record hand position and velocity while generating force feedback at $1 \mathrm{kHz}$ in real time. A six-axis force transducer (ATI Nano 25; ATI Industrial

90 Automation) measured the end-point forces applied by the participant on the handle and was also

91 sampled at $1 \mathrm{kHz}$. Participants were seated in front of the experimental setup and looked at a semi-

92 silvered mirror showing the projection of an LCD screen placed directly above it (Figure 1A) such

93 that visual feedback was presented in the plane of arm movement. An opaque screen below the

94 mirror prevented participants from viewing their hand. The participant grasped the robotic handle

95 using his/her right hand with their arm supported on an air sled allowing frictionless movement.

96 Once the participant was seated in front of the system we measured the shoulder position in system

97 coordinates. To do so, we initially measured the upper arm length and length between elbow joint

98 to the handle center. Next, we placed the hand at the system center location by applying forces

99 using the robot and measured the elbow and shoulder angles. Using these measurements and the

100 inverse kinematics equations of a two-link arm model, we calculated the shoulder location.

101 Participants performed point-to-point reaching movements between a start position and a target 102 position. The participants saw on the screen a cursor (red circle with diameter of $0.7 \mathrm{~cm}$ ) 103 representing their hand. Each trial was started by the robotic system moving the participant's hand

104 to the start position (1.5 $\mathrm{cm}$ diameter gray circle) in which the participants were instructed to wait 105 for a go cue. After a random wait time varying between 0.75 and $1.5 \mathrm{~s}$, a short auditory beep 106 signaled participants to start their movement towards the target (yellow circle with $1.5 \mathrm{~cm}$ 107 diameter). The start and target points were chosen from 16 equally spaced locations on a $10 \mathrm{~cm}$ 108 diameter circle (Figure 1B). Participants were instructed to move with a consistent peak speed of 
$50 \mathrm{~cm} / \mathrm{s}$. To do so, at the end of each movement participants were presented visual feedback about the peak speed of their movement. Peak speed that was within $\pm 8 \mathrm{~cm} / \mathrm{s}$ from the desired peak speed was considered 'good' while a peak speed below this value received a 'too slow' message and above it received a 'too fast' message. In addition, if the participants overshoot the target position by more than $2 \mathrm{~cm}$, they received an 'overshoot' message. For each movement that was considered as a good movement, we increased a score that appeared on the top of the screen by one point.

On each trial, the robot was used to produce one of three virtual environments. The first was a null field (NF), in which the robot was completely passive. For the other two environments, the robot generated forces on the participants' hand during movement. The second virtual environment was a force channel (Scheidt et al., 2001; Milner and Franklin, 2005; Smith et al., 2006), on which we implemented two virtual walls that constrained the participants' hand to move in a straight-line path to the target. The virtual walls were created by generating forces that were perpendicular to the line connecting between the start and target positions. The forces were calculated according to the deviation of the hand position from the straight-line path multiplied by a stiffness coefficient of $4000 \mathrm{~N} / \mathrm{m}$ and the velocity in the movement perpendicular direction multiplied by a damping coefficient of $2 \mathrm{Ns} / \mathrm{m}$. The third type of virtual environment was a force field. During these trials, participants experienced forces acting on their hand that were a function of the hand's instantaneous velocity. The forces were calculated using a scaled curl matrix

$$
\left[\begin{array}{l}
F_{x} \\
F_{y}
\end{array}\right]=B(\dot{x}, \dot{y}) \cdot\left[\begin{array}{l}
\dot{x} \\
\dot{y}
\end{array}\right]=\left[\begin{array}{cc}
0 & 0.16 \\
-0.16 & 0
\end{array}\right] \cdot \cos \left(2 \cdot \arctan \left(\frac{\dot{y}}{\dot{x}}\right)\right) \cdot\left[\begin{array}{c}
\dot{x} \\
\dot{y}
\end{array}\right]
$$

where $F_{x}$ and $F_{y}$ are the forces generated in the $\mathrm{x}$ and $\mathrm{y}$ axis respectively and $\dot{x}$ and $\dot{y}$ are the hand velocities in the $\mathrm{x}$ and $\mathrm{y}$ axis respectively. The traditional curl force field was scaled using a cosine trigonometric function of movement direction. That is, the magnitude and sign of the scaling factor changed according to movement direction, calculated using the inverse tangent function (Figure 1C). For example, when the motion was made only in the x-axis, i.e. horizontal right to left or vice-a-versa, the scaling factor was equal to 1 , or when the motion was made only in the $y$-axis, i.e. moving away or towards the body, the scaling factor was equal to (-1). We define clockwise forces as positive forces and counter-clockwise forces as negative forces. Simulated mean forces that participants experienced can be illustrated as a cosine curve when plotted as a function of movement direction (Figure 1D). 
138 We conducted four experiments using similar experimental protocols. For each experiment, we 139 used three workspaces (differing in their spatial location) in which participants performed the 140 reaching movements; one training workspace and two test workspaces. Each experiment included 141 four experimental phases (Figure 1E). The experiment started with a familiarization phase of 16 142 trials that was performed only in the training workspace. In this phase, participants performed 143 reaching movements from each one of sixteen starting points. The movements were unconstrained 144 reaching movements, i.e. there were no forces applied on participants' hand (NF condition). The 145 second, pre-exposure phase included recording baseline measurements of the movements in the 146 force channel. For this purpose, participants performed 384 movements overall in the training and 147 test workspaces. The movements included both unconstrained reaching movements (NF condition) 148 and movements while the robot generated a virtual force channel between start and target locations 149 (FC condition). Participants performed 4 NF and 4 FC trials for each of the sixteen starting points 150 in each workspace, which means that for each workspace participants performed 128 movements 151 (total 384 movements across the three workspaces). During the third, exposure phase, participants 152 experienced the forces generated by the trigonometric-scaled curl force field (FF condition). 153 Participants performed 15 repetitions from each of the 16 starting points (total of 240 trials), all 154 performed in the training workspace. Finally, after adaptation to the force field, the fourth phase 155 tested generalization of the force field representation in the two test workspaces. During this phase, 156 participants performed 1536 reaching movements in the training workspace on which the force 157 field was applied and an additional 384 movements in the training and test workspaces in which 158 their movements were constrained within the force channel. For each workspace, we had 8 159 repetitions of force channel trials for each of the 16 starting points (total 384 trials). These trials 160 were randomly introduced within the 1536 of force field trials; for every series of 4 force field 161 trials we had one force channel trial. The position of the force channel trial within the series was 162 random with a uniform distribution. Each participant participated in one of four experiments, 163 which differed in the location of the training and test workspaces or had an additional task besides 164 performing reaching movements.

165 Experiment 1. The location of the center of the training workspace was set for each participant so that the participants' shoulder joint was at $50^{\circ}$ and elbow joint was at $90^{\circ}$. The center of the test 
167 workspace 1 was set so with participants' shoulder angle at $50^{\circ}$ and elbow angle at $55^{\circ}$. The center 168 of test workspace 2 was set with the shoulder joint at $75^{\circ}$ and elbow at $45^{\circ}$ (Figure $2 \mathrm{~A}$ ).

169 Experiment 2. The location of the center of the training workspace was set for each participant so 170 that the participants' shoulder joint was at $40^{\circ}$ and elbow joint was at $70^{\circ}$. The center of the test 171 workspace 1 was set so with participants' shoulder angle at $75^{\circ}$ and elbow angle at $85^{\circ}$. The center 172 of test workspace 2 was set with the shoulder joint at $75^{\circ}$ and elbow at $50^{\circ}$ (Figure 2D).

173 Experiment 3. The location of the training and test workspaces were set specifically for each 174 participant and identical to those of experiment 2. However, in this experiment the participant's 175 hand always moved in the training workspace (both training and test workspaces). Movements in 176 the test workspaces were performed using a virtual pole attached to the participants' hand. That is, 177 a virtual green pole linked the red cursor representing the participants' hand to a green cursor 178 located in the test workspace. This green cursor then moved with this fixed offset to the 179 participant's hand, as the participant's hand moved. We instructed participants to move the green 180 circle between the start and target instead of their hand. The length and orientation of the pole was 181 set based on the vector connecting the center of the training and test workspaces (Figure 2G). In 182 order to move between a particular set of start and target points in the test workspace, participants 183 had to perform similar movement between the same start and target points in the training 184 workspace with their hand. Thus, the joint trajectory was similar regardless of whether it was 185 performed in the training or test workspaces.

186 Experiment 4. This experiment was similar to experiment 1 or 2 but with different workspace 187 locations (Figure 2J). The center of the training workspace was set for each participant so that their 188 shoulder joint was at $55^{\circ}$ and elbow joint was at $75^{\circ}$. The center of test workspace 1 and 2 was set 189 such that the shoulder and elbow joints were oriented at $\left[30^{\circ}, 100^{\circ}\right]$ and $\left[80^{\circ}, 50^{\circ}\right]$ respectively. 190 These workspace locations kept the hand orientation constant while the arm configuration varied. 
A

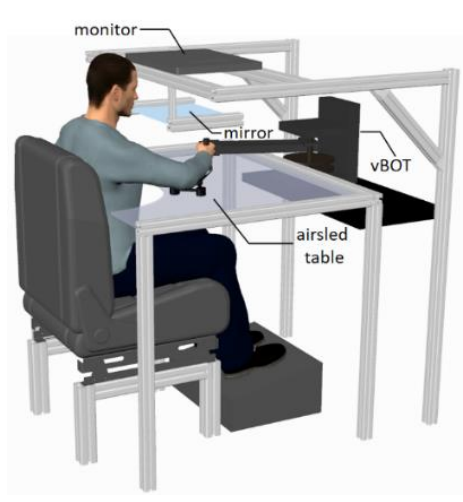

B

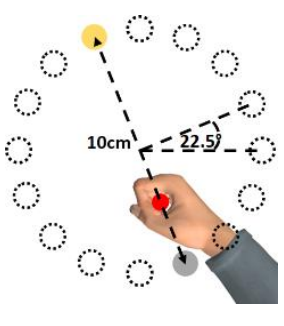

$\mathrm{E}$
$\mathrm{C}$

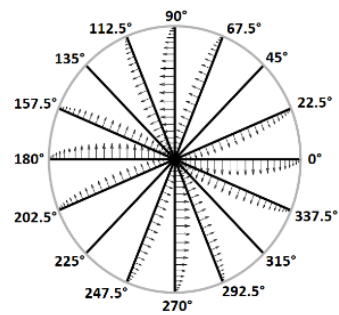

$\mathrm{D}$

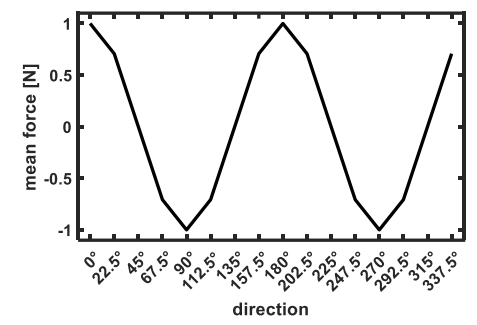

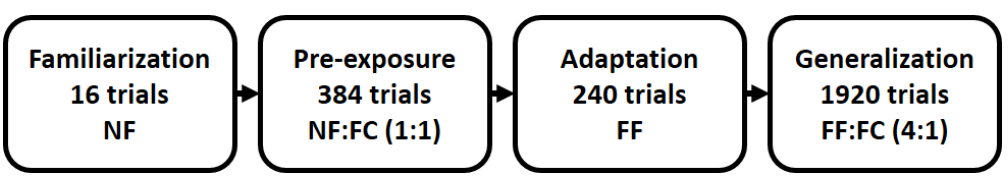

Figure 1: Experimental setup.

A) Participants sat in front of a robotic manipulandum system (vBOT) while grasping the handle of the robot with their right hand. The participants' arm was supported by an airsled system that reduced friction during movement. The virtual environment was projected on a mirror from a monitor mounted above the movement space.

B) Display of the virtual workspace. The participants' hand was represented using a red cursor that was aligned with the hand position. The task was to move from the start point (grey circle) to the target point (yellow circle). The start and target points were always located on the diameter of a $10 \mathrm{~cm}$ diameter circle. There were 16 possible starting points spaced evenly on the circumference of the circle. On each trial only one set of start and target points were presented.

C) Endpoint force profiles as a function of movement direction. The forces were calculated using a scaled curl force field (equation 1).

D) Cosine representation of the mean forces of the scaled force field as a function of movement direction. Clockwise forces were set as positive and counterclockwise forces were set as negative.

E) Experimental protocol. Initial familiarization phase included 16 trials in which no force applied on the hand (NF condition). The pre-exposure phase included unconstrained movements (NF condition) and movements in a force channel (FC condition), with a ratio of 1:1 in all three workspaces. In the adaptation phase, participants performed the movements under the force field perturbation (FF condition) only in the training workspace. In the last phase we tested generalization using force channel trials (FC) that were introduced randomly at the training and test workspaces while participants kept moving under force field perturbations (FF) only in the training workspace. The ratio between force field and force channel trials was $4: 1$. 
193 Data analysis was performed offline using Mathworks Matlab 2018b®.

194 Maximum Perpendicular Error (MPE). On each null field or force field trial the maximum distance 195 between the trajectory and the straight line joining the start and target points was calculated as a 196 measure of kinematic error.

197 Force Compensation. The force compensation was calculated during the generalization phase for 198 the training and test workspaces on the force channel trials. Force compensation in the training 199 workspace indicates the degree of adaptation of individual participants to the force field whereas 200 force compensation in the test workspaces demonstrates how the learned forces are generalized in 201 space. Force compensation was calculated as the regression slope between the perfect force 202 compensation signal, the independent variable, and the recorded force signal, the dependent 203 variable (Smith et al., 2006). The recorded force profile for each channel trial was identified 204 between the start and end of the movement (first and last point at which velocity exceeded 5\% 205 maximum velocity). The perfect compensation force profile was determined as the force required 206 for perfect compensation of movement in an unscaled curl force field and was calculated by 207 multiplying the unscaled curl force field matrix by the velocity vector, $208\left[\begin{array}{lllll}0 & 0.16 ; & ; & -0.16 & 0\end{array}\right] \cdot[\dot{x} ; \dot{y}]$. Here we compare the recorded force profile with forces 209 computed using an unscaled version of the curl force field and not the scaled curl force field for 210 three reasons. First, for some directions there was no force perturbations (e.g. moving at a direction 211 of $45^{\circ}$ ), even the smallest force participants generate during force channel trials will result in an 212 infinite slope value. The second reason is to capture the direction of the forces that participant 213 generated, i.e. clockwise or counter-clockwise. Regression against always positive values of the 214 perfect force profile allows us to identify in which directions the participants generated positive or 215 negative forces. This allows us to identify any shift in the force compensation profiles when plotted 216 as a function of movement direction. Third, by calculating the regression against the same value 217 across all directions allows us to identify the force magnitude participants adapted to as a function 218 of movement direction. Once we calculated the force compensation measurement for each 219 movement in the force channel, we averaged across the eight repetitions for each movement 220 direction and repeated this for all 16 directions and each workspace. The resulting pattern of the 221 force compensation measure across all movement directions resembles a cosine function. To 
222 quantify phase differences between the scaled force field and the force compensation curves

223 extracted from movements in the training and test workspaces, we calculated the cross-correlation

224 between these cosine signals. That is, the cross-correlation provided a measure for the shift of each

225 force compensation curve from the designed scaled force field.

\section{Prediction of Generalization Models}

227 We considered three different coordinate frames in which the motor system could represent the 228 learned dynamics. By examining the cosine shape of the learned dynamics as a function of 229 movement direction (Figure 1D), we can distinguish in which coordinate system participants 230 represented the forces.

231 The first coordinate system we considered is the Cartesian coordinate system. For this 232 representation, the forces are invariant with respect to the arm orientation. In such case, 233 participants will exhibit similar forces to the learned forces for each one of the test workspaces 234 (equation 1), resulting in a similar cosine signal of generalized forces as a function of movement 235 direction.

236 The second coordinate system we considered is the joint-based coordinate system (Shadmehr and 237 Mussa-Ivaldi, 1994; Malfait et al., 2002). For this representation, the generalized forces exhibited 238 in the test workspaces should differ from the learned forces since the arm configuration has 239 changed. That is, for experiment 1, 2, and 4 we expect a change in the cosine function describing 240 the generalized forces. To calculate the expected generalized forces profile, we used a two-link 241 arm model in which $\theta=\left[\theta_{s}, \theta_{e}\right]$ represents a vector of the shoulder and elbow joints values. Initially,

242 we need to derive the learned dynamics in joint coordinate system and then derive the expected 243 generalized forces. In joint representation, the learned forces are represented as joints torques using 244 the Jacobian which form the relation between the two variables, $\tau=J_{\text {train }}^{T} \cdot \boldsymbol{F}$, where $\boldsymbol{F}=\left[F_{x}, F_{y}\right]$

245 is a vector of the end-point forces, $\tau=\left[\tau_{s}, \tau_{e}\right]$ is a vector of joints torques, and $J_{\text {train }}$ is the arm's

246 Jacobian matrix for the training configuration. Similarly, the hand velocities are represented using

247 joint angular velocity and the Jacobian matrix, $\boldsymbol{v}=J_{\text {train }} \cdot \dot{\boldsymbol{\theta}}$, where $\boldsymbol{v}=[\dot{x}, \dot{y}]$ is the hand velocity 248 vector. Thus, using the end-point forces generated by the force field (equation 1) we derived the 249 learned joint torques 


$$
\boldsymbol{\tau}=J_{\text {train }}^{T} \cdot \boldsymbol{F}=J_{\text {train }}^{T} \cdot B(\boldsymbol{v}) \cdot \boldsymbol{v}=J_{\text {train }}^{T} \cdot B\left(J_{\text {train }} \cdot \dot{\boldsymbol{\theta}}\right) \cdot J_{\text {train }} \cdot \dot{\boldsymbol{\theta}}
$$

250 The expected forces during movement in the test workspaces can be compute using the learned 251 torques and the arm's Jacobian for the test configuration.

$$
\begin{aligned}
\boldsymbol{F} & =J_{\text {test }}^{-T} \cdot \boldsymbol{\tau} \\
& =J_{\text {test }}^{-T} \cdot J_{\text {train }}^{T} \cdot B\left(J_{\text {train }} \cdot \dot{\boldsymbol{\theta}}\right) \cdot J_{\text {train }} \cdot \dot{\boldsymbol{\theta}} \\
& =J_{\text {test }}^{-T} \cdot J_{\text {train }}^{T} \cdot B\left(J_{\text {train }} \cdot J_{\text {test }}^{-1} \cdot \boldsymbol{v}\right) \cdot J_{\text {train }} \cdot J_{\text {test }}^{-1} \cdot \boldsymbol{v}
\end{aligned}
$$

252 The third coordinate system we considered is the object-based coordinate system (Berniker et al., 253 2014). For this representation, the motor system relates the dynamics to a grasped object. When 254 moving outside of the training workspace, the expected forces are calculated according to the 255 orientation of the held object, which is rotated with the hand orientation. For example, if we learned 256 a force profile while moving straight away from the body and we now moved to another location 257 in which the hand was rotated by an angle $\theta$ in external space, the learned profile is expected to 258 appear at a direction which is rotated by $\theta$. That is, initially, based on the velocity vector, we can 259 find the learned force profile by rotating back to the original training workspace $260 \boldsymbol{F}_{\text {train }}=B\left(R^{-1}(\theta) \cdot v\right) \cdot R^{-1}(\theta) \cdot v$, where $\mathrm{R}$ is a rotation matrix. Then, we rotate these forces to the 261 test workspace:

$$
\boldsymbol{F}_{\text {test }}=R(\theta) \cdot \boldsymbol{F}_{\text {train }}
$$

262 The predictions of these models for each of four experiments are depicted in Figure 2. We 263 simulated the expected forces according to the models for the two test workspaces using the 264 different arm postures. For experiment 1 (Figure 2B and 2C), using a Cartesian based 265 representation, the expected forces do not change when switching between workspaces. Thus, the 266 force compensation profile is similar to the profile of the force field for both test workspaces 1 and

267 2. In the case of a joint-based representation, since the arm posture changes between workspaces, 268 the force compensation profile for the test workspace changes compared with the learned force 269 field. For test workspace 1, there is a leftward shift of the force compensation curve compared 270 with the learned force field curve for some of the directions. For test workspace 2 there is a leftward 271 shift for some parts of the signal and a rightward shift for the rest of the signal compared with the 272 learned force field. Similar to the joint-based representation, for object-based representation, the 273 force compensation profiles change as the orientation of the hand is changed between workspaces. 
274 For both workspaces, the curves are shifted to the left with an increased leftward shift for test 275 workspace 1. Thus, leftward shift of the force compensation curve for the test workspace 1 276 compared to the learned forces curve would suggest that the representation does not rely on a 277 Cartesian based coordinate system. Similarly, leftward shift of the curve for test workspace 2 278 would suggest that the representation relies more on an object-based representation while a 279 rightward shift would suggest that the representation relies more on a joint-based representation.

280 For experiment 2 (Figures 2E and 2F), the Cartesian based representation again predicts that the 281 expected force profile in the test workspaces will be the same as the adapted force profile in the 282 training workspace. In the case of a joint-based representation or an object-based representation, 283 both predict a shift to the right with an increased rightward shift for test workspace 1.

284 For experiment 3 (Figures $2 \mathrm{H}$ and 2I), both the Cartesian based and joint-based representations 285 predict that the force compensation curves do not change or shift compared with the learned force 286 field curve since the hand posture does not change between the training workspace and test 287 workspaces. However, for the object-based representation, since the orientation of the pole is 288 different between the training and test workspaces, the predictions of the force compensation curve 289 are shifted to the right, more strongly so for test workspace 1. Thus, for this experiment, a 290 rightward shift of the force compensation curves would suggest that representation relies more on 291 an object-based representation.

292 For experiment 4 (Figures 2J and 2K), the Cartesian based representation again predicts that the 293 expected force profile in the test workspaces will be the same as the adapted force profile in the 294 training workspace. Similarly, the object-based representation predicts the same expected force 295 profile as hand orientation does not change between the training and test workspaces. However, 296 since the arm configuration changes between these workspaces, the joint-based representation 297 predicts alterations in the expected force profiles. For test workspace 1, joint-based representation 298 predicts a shift to the left of the curve for some movement directions while for test workspaces 2 299 the prediction is a rightward shift of the curve. If instead there are small or no shifts of the curves 300 then this would argue against a joint-based representation.

301 Alongside the individual model predictions we also considered that representation might be based 302 on a mixture of models (Berniker et al., 2014). That is, we considered that the expected forces in 
303 the test workspaces are calculated as a weighted sum of the expected force values of the individual

304 models:

$$
\boldsymbol{F}=k_{c} \cdot \boldsymbol{F}_{\text {Cartesian }}+k_{j} \cdot \boldsymbol{F}_{\text {joints }}+k_{o} \cdot \boldsymbol{F}_{\text {object }} .
$$

305 For this model, we searched for the optimal weights, $k_{c}, k_{j}, k_{o}$, such that the squared error

306 between the mean force profile of participants and the weighted predicted force, calculated using 307 the simulations, is minimized. For this optimization problem, we constrained the weights to 308 positive values and set the sum of the weights equal to one. The constraint on the weighted sum is 309 a result of assuming that the expected forces in the test workspaces will have the same magnitude 310 as in the training workspace. However, previous studies suggested that the magnitude of the 311 generalized forces might decay as we are moving away from the training workspace (Gandolfo et 312 al., 1996; Berniker et al., 2014). Thus, we also considered a mixture model with a decay factor by 313 constraining the sum of weights to be equal or less than one.

314 Overall, we considered 14 models; 6 individual models with or without decay, and 8 mixture 315 models covering all combinations of the individual models with and without decay. To compare 316 the performance of the models in explaining the mean generalized force profiles, we used the 317 Bayesian information criterion (BIC):

$$
B I C=-2 \cdot \log (L)+k \cdot \log (n)
$$

318 where $\log (L)$ is the logarithm of the model likelihood, $\mathrm{k}$ is the number of degrees of the model (1 319 for individual models and 2 or 3 for mixture models), and $n=32$ is the total number of data points. 320 We fitted the models separately for the results of each experiment and afterwards calculated the 321 BIC for each model where smaller BIC value indicates that the model is a better fit to the data. 322 This allowed us to identify the best models that explain the mean generalized force profiles. For 323 these best-fit models, we then repeated the fitting to the profiles of each participant. This individual 324 fitting serves as an indication for the variance of the optimal weights fitted to the mean force 325 profile. In addition, to examine the overall fit of the models, we fitted the models to the entire 326 dataset, i.e. all four experiments, followed by a BIC calculation for each model. 


\section{Statistical Analysis}

328 To compare the phase differences between the scaled force field curve and the force compensation

329 curves we fitted a one-way repeated measures ANOVA model with phase difference as the

330 dependent factor and the workspace as the within-subject independent factor (3 levels; difference

331 between the training workspace, workspace 1 and workspace 2 and the scaled force field). We

332 repeated this test for all experiments. When the phase factor was found to be statistically

333 significant, we used post-hoc tests with a Bonferroni correction for multiple comparisons.

334 Statistical significance was determined at the 0.05 threshold in all tests. 
A

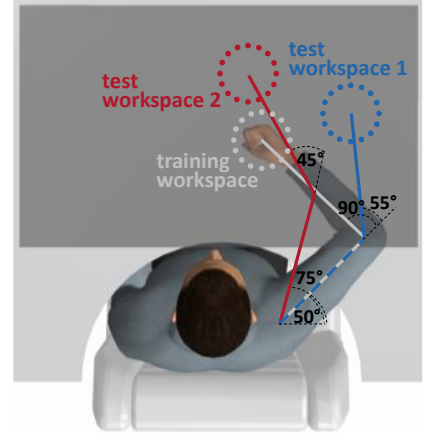

$\mathrm{D}$

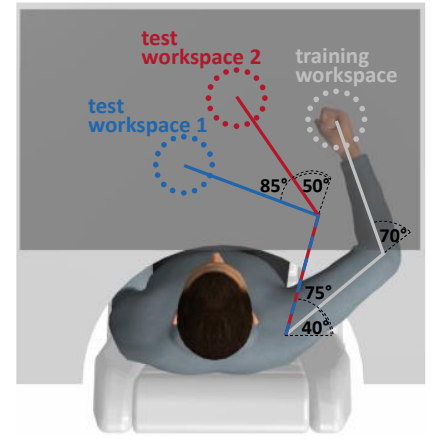

G

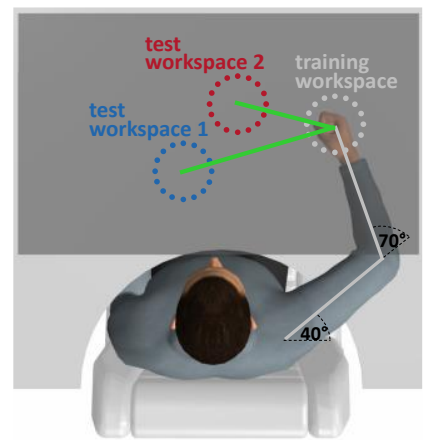

$\mathrm{J}$

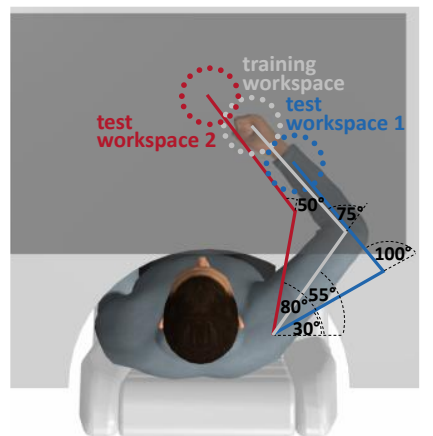

B

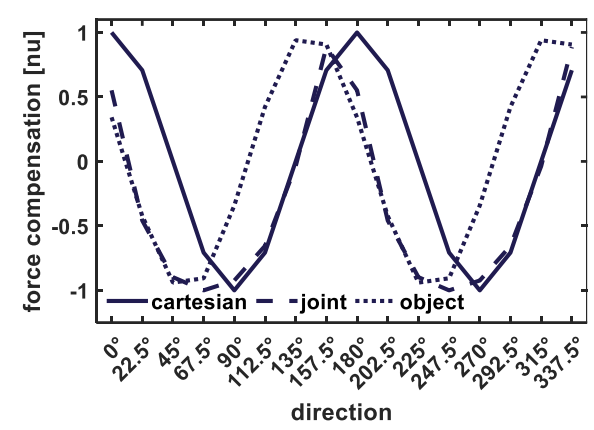

$\mathrm{E}$

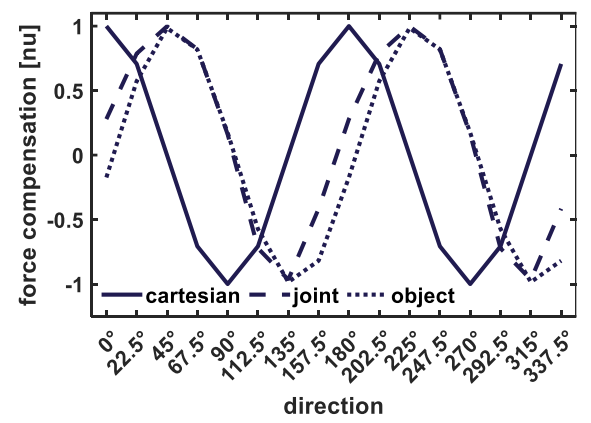

$\mathrm{H}$

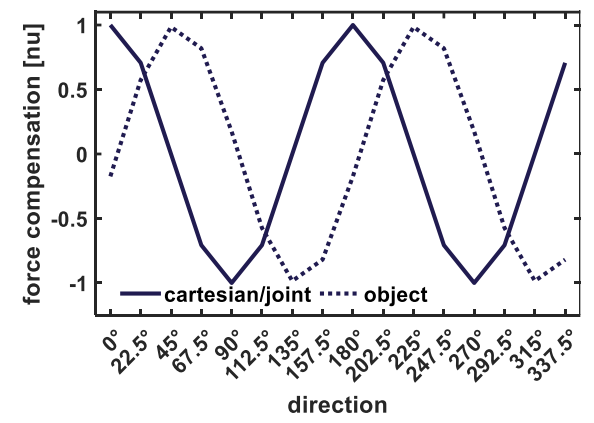

$\mathrm{K}$

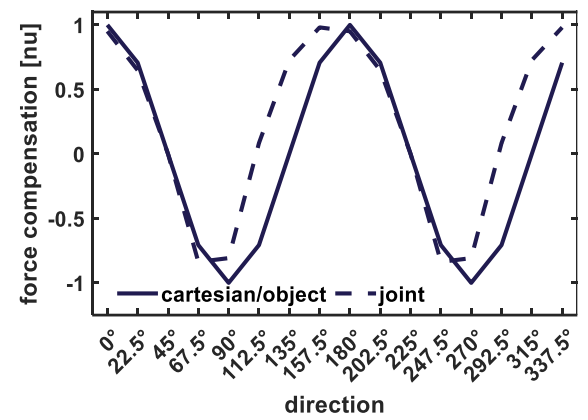

$\mathrm{C}$

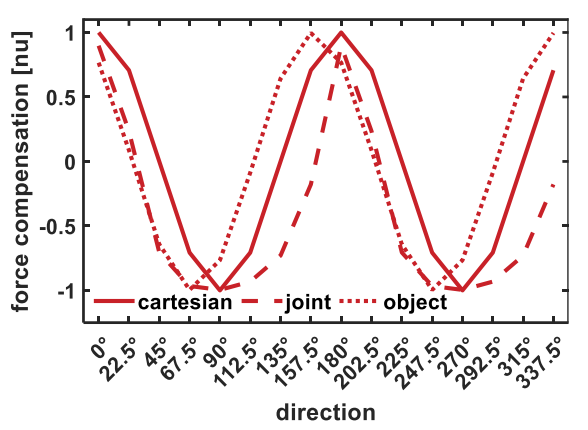

F

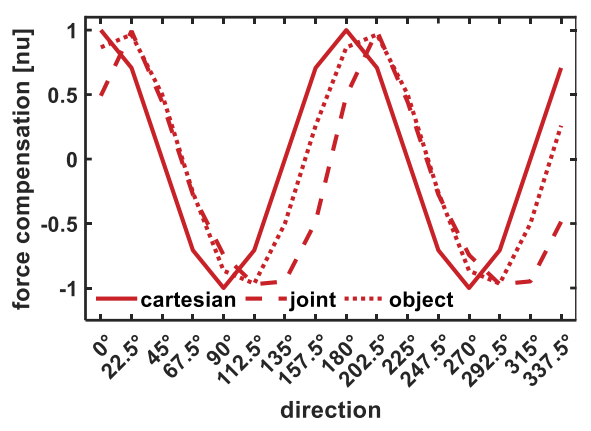

I

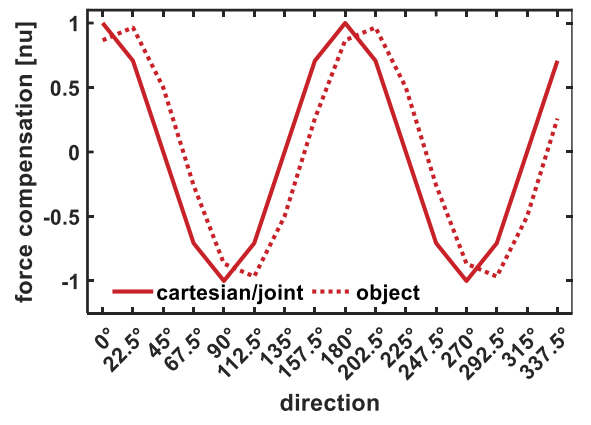

$\mathrm{L}$

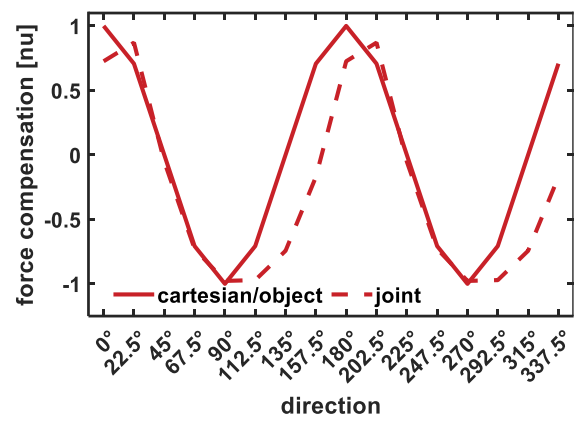

Figure 2: Workspaces locations and force generalization predictions of the models. A) Workspaces locations for experiment 1. Participants adapted to the scaled curl force field in the training workspace (grey) while force generalization was tested in test workspace 1 (blue) and test workspace 2 (red). Each workspace location was set based on predefined values of shoulder and elbow joint angles and the measured length of the upper and forearm. Only the start and target locations for the current movement were displayed on each trial. B) Force compensation predictions for test workspace 1 in experiment 1 for Cartesian (solid line), joint (dashed line), or object (dotted line) representations of the dynamics. C) Force 
compensation predictions for test workspace 2 in experiment 1 . D) Workspaces locations for experiment 2. E\&F) Force compensation predictions for test workspaces in experiment 2. G) Workspace locations for experiment 3 . The participants always moved in the training workspace and the generalized forces were measured during movement of the endpoint of a pole (visually attached to the hand) in the same test workspaces as in experiment 1 . H\&I) Force compensation predictions for test workspaces in experiment 3. J) Workspace locations for experiment 4. K\&L) Force compensation predictions for test workspaces in experiment 3.

Across all test workspaces and experimental conditions, the Cartesian based representation predicts the same generalized force pattern as the learned force field. When the arm configuration differs between training and test workspaces, the joint-based representation predicts a shift to the right (panels E, F and L), left (panels B and K) or both (panel C) of the generalized force pattern with respect to the learned force field. When the hand orientation differs between training and test workspaces, the object-based representation predicts a shift to the right (panels E, F, H, and I) or left (panels B and C) of the generalized force pattern. 


\section{Results}

337 In experiment 1, participants adapted to the scaled curl force field as indicated by both the 338 reduction in maximum perpendicular error (Figure 3A) and the increase in predictive force 339 compensation profile in the training workspace (Figure 3B, grey curve) that resembles the cosine 340 tuning of the scaled force field (Figure 1D). The generalized force compensation in test workspaces 3411 and 2 also exhibited a cosine pattern; however, it was shifted with respect to the curve of the 342 training workspace or the curve representing the force field. For the curve calculated based on 343 movements in the training workspace (Figure 3B and 3C, grey curve) we did not find a shift in 344 respect to the original curve describing the force field $\left(-0.01 \pm 0.03^{\circ}\right.$, mean \pm STD $)$. For the curve 345 calculated based on movements in test workspace 1 (Figure 3B, blue curve), we found a leftward 346 shift of $-23 \pm 4.8^{\circ}$ in respect to the original curve describing the force field. For the curve calculated 347 based on movements in test workspace 2 (Figure 3B, red curve) we again found a leftward shift of 348 the curve, however, this shift $\left(-6.3 \pm 4.8^{\circ}\right)$ was smaller compared with the shift we found for test 349 workspace 1 . These observations were confirmed using a repeated measures ANOVA that showed 350 a statistically significant effect of the workspace condition $\left(F_{2,14}=115.6, p<0.001\right)$. Post-hoc analysis showed that the training curve was not statistically significantly different from the force

352 field curve $\left(\mathrm{t}_{7}=1, p=0.35\right)$. However, both curves for workspace 1 and workspace 2 were 353 statistically significant different from the force field curve (both $\mathrm{t}_{7}>3.7, \mathrm{p}<0.01$ ), from the training 354 workspace curve (both $\left.\mathrm{t}_{7}>3.72, p<0.05\right)$ and from each other $\left(\mathrm{t}_{7}=13.4, p<0.001\right)$.

355 These trends match the trends predicted by a mixture model that rely mostly on the object-based 356 representation. The object-based representation predicts a leftward shift of the curve based on test 357 workspace 1 (Figure 3C upper panel, dot curve) and a smaller leftward shift of the curve based on 358 test workspace 2 (Figure 3C bottom panel, dot curve). Importantly, for some of the directions, only 359 the simulation based on the object-based representation predicted the leftward shift we observed 360 for the curve of workspace 1 and 2 . When fitted to the data, we found that a mixture model 361 combining the Cartesian, joint- and object-based representation $(\mathrm{C}+\mathrm{J}+\mathrm{O})$ with a decay factor best 362 fits the experimental results (Figure 3D). The optimal weights of this model were 0.31 for the 363 contribution of the object-based representation and 0.22 and 0.1 for the contribution of the joint364 based and Cartesian based representations respectively. Another mixture model that fits the data 365 was a mixture of the object- and joint- based representations $(\mathrm{J}+\mathrm{O})$ with a decay factor. For this 


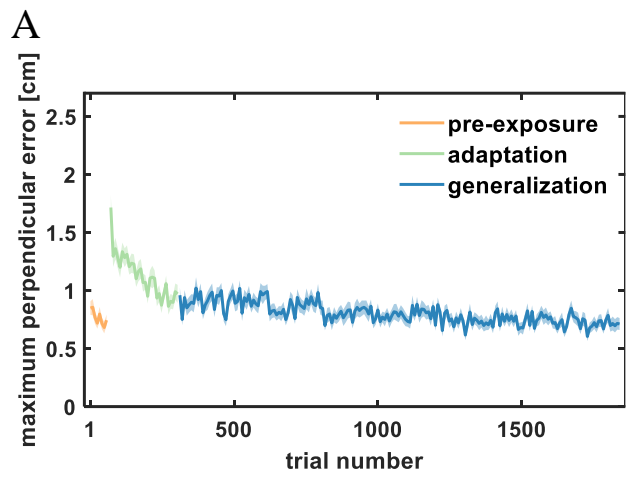

B

$\mathrm{C}$
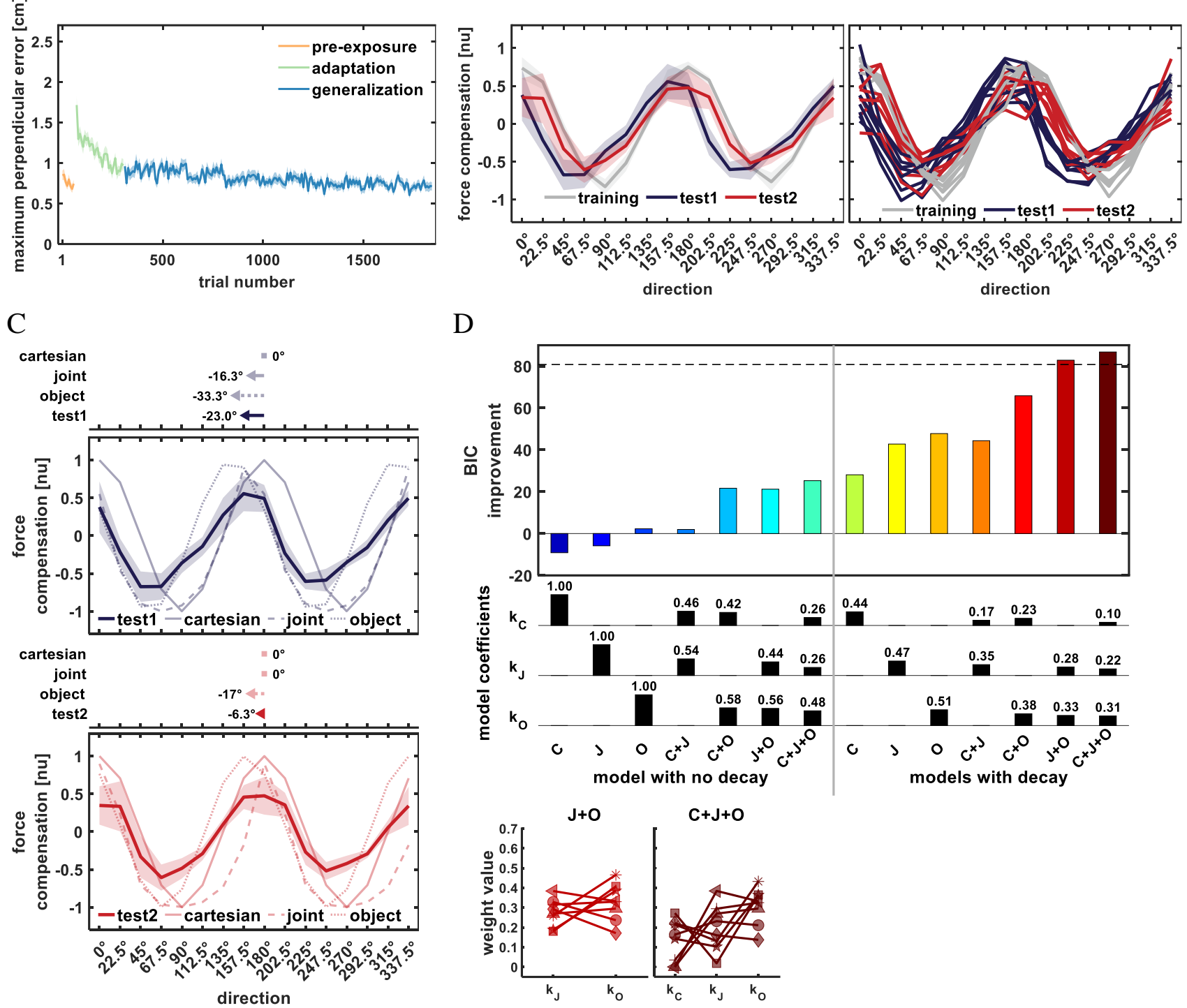

$\mathrm{D}$
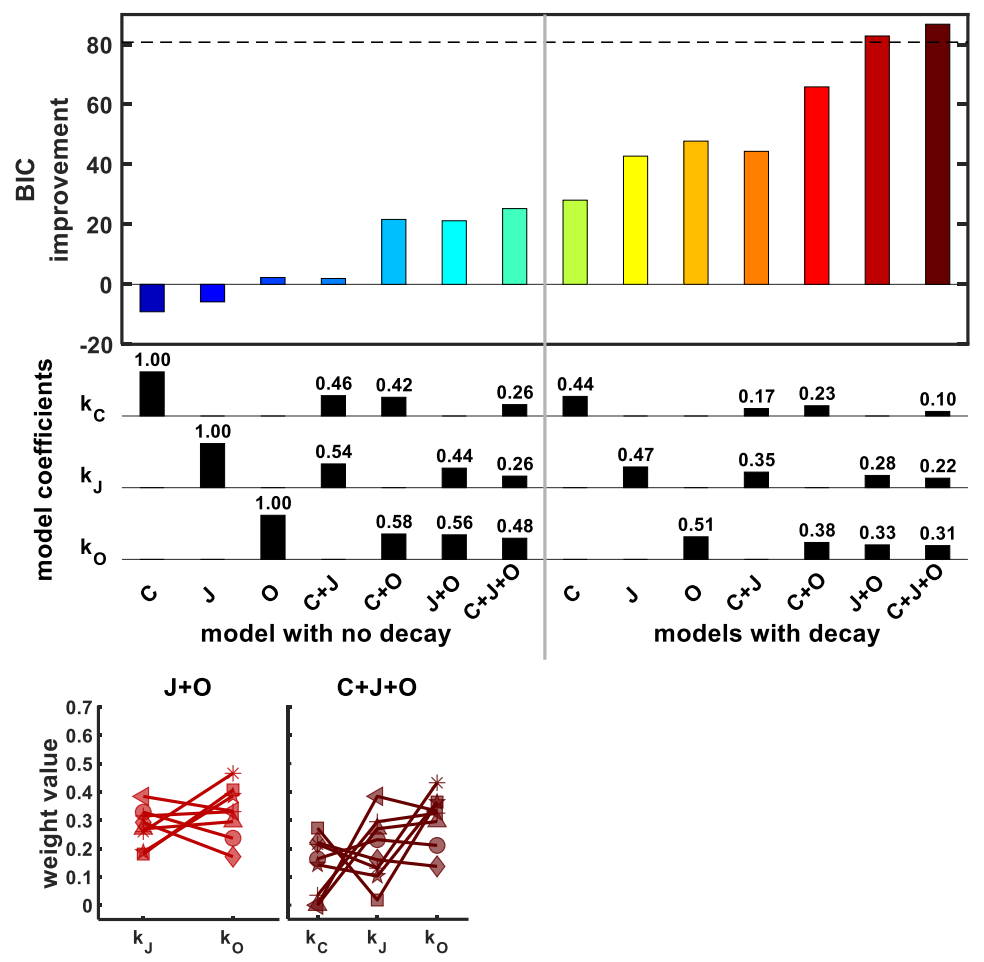

Figure 3: Results of experiment 1.

A) Mean maximum perpendicular error (MPE) and standard error (shaded area) across participants during pre-exposure phase (orange line), adaptation phase (green line), and generalization phase (blue line) of movements performed in the training workspace.

B) Left panel, mean force compensation profiles. Participants were able to adapt to the scaled force field as evident by the force compensation profile in the training workspace (grey curve) and showed a rightward shift of the curve for the test workspace 2 (red curve) and more so for test workspace 1 (blue curve). Right panel, Individual force compensation profiles for each participant in the training workspace (grey curve), workspace 1 (blue curve), and workspace 2 (red curve).

C) Upper panel, mean force compensation profile for workspace 1 (dark blue solid line) and Cartesian (light blue solid line), joint- (light blue dashed line), and object- (light blue dotted line) based model predictions (same as Fig. 2B). Bottom panel, mean force compensation profile for workspace 2 (dark red solid line) and 
Cartesian (light red solid line), joint- (light red dashed line), and object- (light red dotted line) based model predictions (same as Fig. 2C). Arrows at the top of the panels indicate the shift of the mean force compensation profile and the models predictions in respect to the original curve describing the force field.

D) Upper panel, BIC Improvement for each of the models relative to no generalization (that is, a model in which the force is zero at all movement directions). Dashed line shows the cutoff for models that are not considered distinguishable in terms of their performance from the best model $(\mathrm{C}+\mathrm{J}+\mathrm{O}$ model $)$. Middle panel, the fitted coefficients for each model. C-Cartesian, J- joint, O- object. Bottom panels are the fitted coefficients for the best models $(\mathrm{J}+\mathrm{O}$ and $\mathrm{C}+\mathrm{J}+\mathrm{O})$ when fitting the models to individual participant data, indicating the variance of the fitted coefficients values.

In experiment 2, we found similar adaption trend to the scaled curl force field as seen in the reduction in maximum perpendicular error (Figure 4A). The force compensation curve in the training workspace resembles the cosine tuning of the scaled force field (Figure 4B, grey curve), and we found similar pattern for the force compensation in test workspaces 1 and 2 but for this experiment they were shifted to the right with respect to the curve of the training workspace or the curve representing the force field. Similar to experiment 1, the curve calculated based on movements in the training workspace was not shifted in respect to the original curve describing the force field $\left(-1 \pm 2.45^{\circ}\right.$, mean \pm STD). For the curve calculated based on movements in test workspace 1 (Figure 3B, blue curve), we found a rightward shift of $34.5 \pm 1.2^{\circ}$ in respect to the original curve describing the force field. For the curve calculated based on movements in test workspace 2 (Figure 3B, red curve) we again found a significant rightward shift of the curve, however, this shift of $15.4 \pm 4.4^{\circ}$ was smaller compared with the shift we found for test workspace 1. These observations were confirmed using a repeated measures ANOVA that showed a statistically significant effect of the workspace condition $\left(\mathrm{F}_{2,14}=306.8, \mathrm{p}<0.001\right)$. Post-hoc analysis showed that the training curve was not statistically significantly different from the force field curve

$384\left(\mathrm{t}_{7}=1.1, p=0.3\right)$. However, both curves for workspace 1 and workspace 2 were statistically 385 significantly shifted from the force field curve (both $\mathrm{t}_{7}>9.8, \mathrm{p}<0.001$ ), from the training workspace curve (both $\left.\mathrm{t}_{7}>9.4, p<0.001\right)$ and from each other $\left(\mathrm{t}_{7}=12.5, p<0.001\right)$.

387 These trends match the predictions of the joint- and object-based representations. Both 388 representations predicted a rightward shift of the curve based on test workspace 1 (Figure 4C upper 389 panel) and a smaller rightward shift of the curve based on test workspace 2 (Figure 4C bottom 390 panel). When fitted to the data, we found that a mixture model of all three representations $(\mathrm{C}+\mathrm{J}+\mathrm{O})$ 391 with a decay factor best fits the experimental results (Figure 4D). The optimal weights of the 392 Cartesian, joint- and object-based representations were 0.14, 0.28, and 0.28 respectively. Another 
393 mixture model that fits the data was a mixture of the Cartesian (optimal weight, 0.16) and the

394 object-based representation (optimal weight, 0.53) with a decay factor.

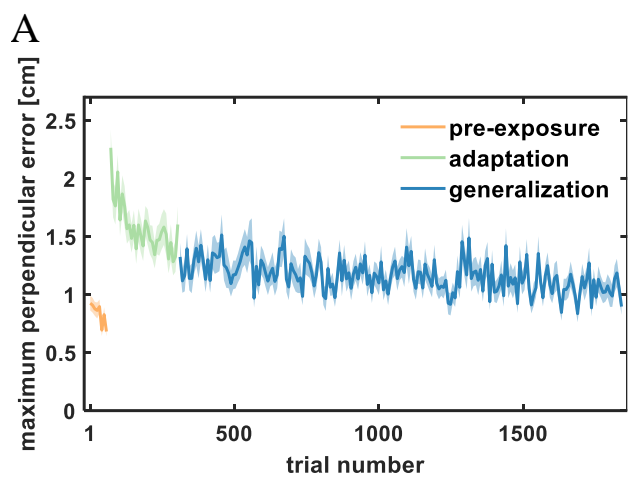

B

$\mathrm{C}$

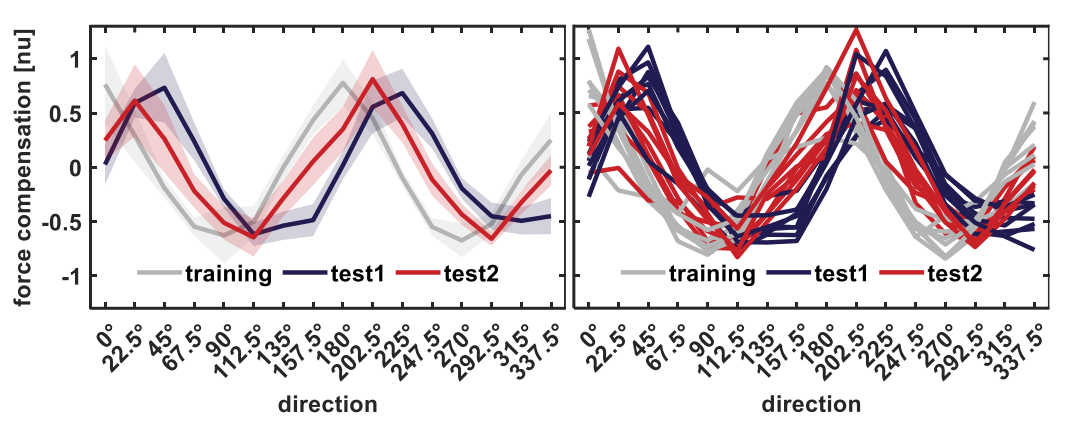

$\mathrm{D}$
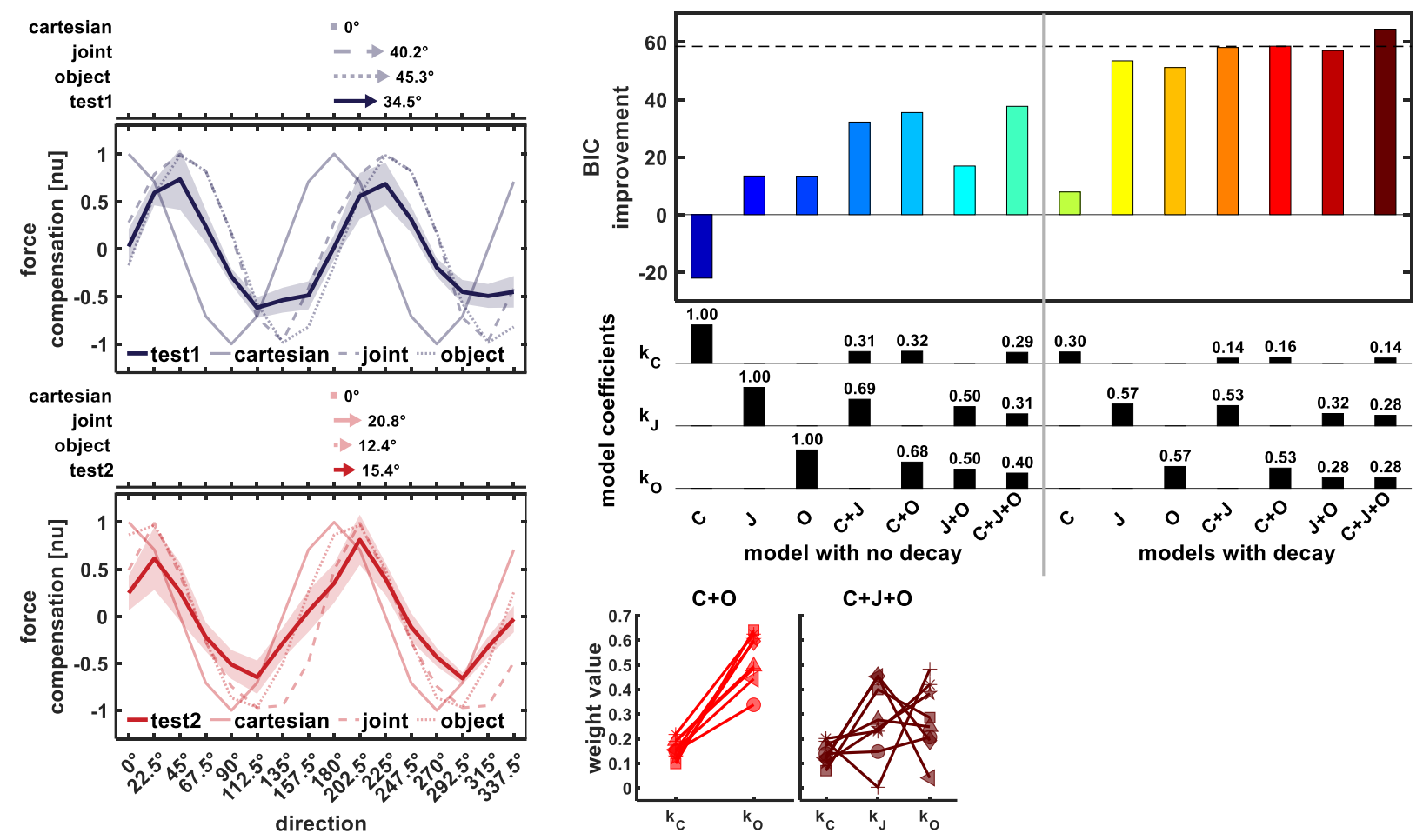

Figure 4: Results of experiment 2. All notations are similar to Figure 3.

A) Mean maximum perpendicular error (MPE).

B) Left panel, mean force compensation profiles in the training and test workspaces. Right panel, individual force compensation profiles in the training and test workspaces.

C) Upper panel, mean force compensation profile for workspace 1 and model predictions (same as Fig. 2E). Bottom panel, mean force compensation profile for workspace 2 and model predictions (same as Fig. 2F). Arrows at the top of the panels indicate the shift of the mean force compensation profile and the models predictions in respect to the original curve describing the force field.

D) Upper panel, BIC Improvement for each of the models relative to no generalization. Dashed line shows the cutoff for models that are not considered distinguishable in terms of their performance from the best model $(\mathrm{C}+\mathrm{J}+\mathrm{O}$ model $)$. Middle panel, the fitted coefficients for each model. C-Cartesian, J-joint, O- object. Bottom panels are the fitted coefficients for the best models $(\mathrm{C}+\mathrm{O}$ and $\mathrm{C}+\mathrm{J}+\mathrm{O})$ when fitting the models to individual participant data. 
In experiment 3, after adaptation to the scaled force field in the training workspace (Figure 5A), participants had to move the tip of a pole between start and target positions in the test workspaces.

397 That is, the arm posture did not change between the adaptation and generalization parts of the 398 experiment. In this case, both the Cartesian and joint-based representations predict that the force 399 compensation curve for the test workspaces will not shift compared with the force compensation 400 curve for the training workspace. Contrary to these predictions, the object-based representation 401 predicts a rightward shift for both workspace 1 and 2, with a stronger shift for workspace 1 (Figure $4022 \mathrm{H}$ and 2I, dotted curves). We found a rightward shift of the curve for both test workspaces with 403 respect to the training workspace. For the curve calculated based on movements in the training 404 workspace (Figure 5B, grey curve) we did not find a shift with respect to the original curve 405 describing the force field $\left(-0.05 \pm 0.14^{\circ}\right.$, mean \pm STD $)$. For the curve calculated based on movements 406 in test workspace 1 , we found a rightward shift of $17 \pm 5.7^{\circ}$ (Figure $5 \mathrm{~B}$, blue curve). For the curve 407 calculated based on movements in test workspace 2 we again found a rightward shift of the curve, 408 however, this shift $\left(4.5 \pm 3.6^{\circ}\right)$ was smaller compared with the shift we found for test workspace 1 409 (Figure 5B, red curve). These observations were confirmed using a repeated measures ANOVA 410 that showed a statistically significant effect of the workspace condition $\left(F_{2,14}=66.0, p<0.001\right)$. Post411 hoc analysis showed that the training curve was not statistically significantly different from the 412 force field curve $\left(\mathrm{t}_{7}=1, p=0.351\right)$. For test workspaces 1 and 2 , we found a statistically significant 413 shift of the force compensation curves of these workspaces from the force field curve (both $\mathrm{t}_{7}>3.5$, $414 \mathrm{p}<0.05$ ), from the training workspace force compensation curve (both $\mathrm{t}_{7}>3.6, \mathrm{p}<0.05$ ), and from 415 each other $\left(\mathrm{t}_{7}=9.96, p<0.001\right)$.

416 Similar to the results of experiment 2, we found that the object-based model predicts the rightward 417 shifts of the force compensation curves of test workspaces 1 and 2 (Figure 5C). The best model 418 that capture these results is a mixture model combining the Cartesian or joint-based representations 419 with the object-based representation $(\mathrm{C}+\mathrm{O}$ or $\mathrm{J}+\mathrm{O})$ with a decay factor (Figure 5D). The optimal 420 weights of these models were 0.24 for the contribution of the object-based representation and 0.39 421 for the contribution of the Cartesian or joint-based representations. Since the Cartesian and joint422 based representations predict the same generalized forces curves we cannot distinguish between 423 them, however, the best fit to the experimental data is a result of combining either of these 424 representations with the object-based representation. Other models that can explain the data were 425 Cartesian based model (C), joint-based model (J), and a mixture of all three representations 
$426(\mathrm{C}+\mathrm{J}+\mathrm{O})$. This last mixture model had same weight value $(0.24)$ for the object-based representation 427 as in the $\mathrm{C}+\mathrm{O}$ or $\mathrm{J}+\mathrm{O}$ models and an equally divided weight for the Cartesian and joint-based 428 representation (0.195) since these two representations predicted similar curves for this experiment. 429 The individual Cartesian or joint-based models were also not distinguishable from the best model 430 since the rightward shifts were not as strong as in experiment 2. 

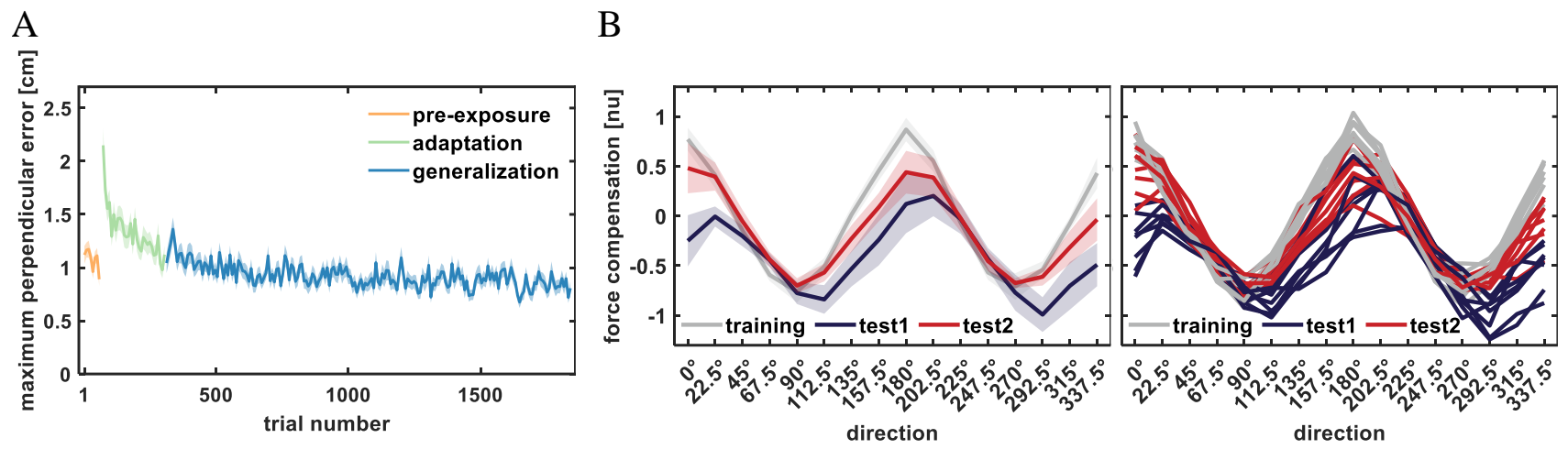

C

$\mathrm{D}$
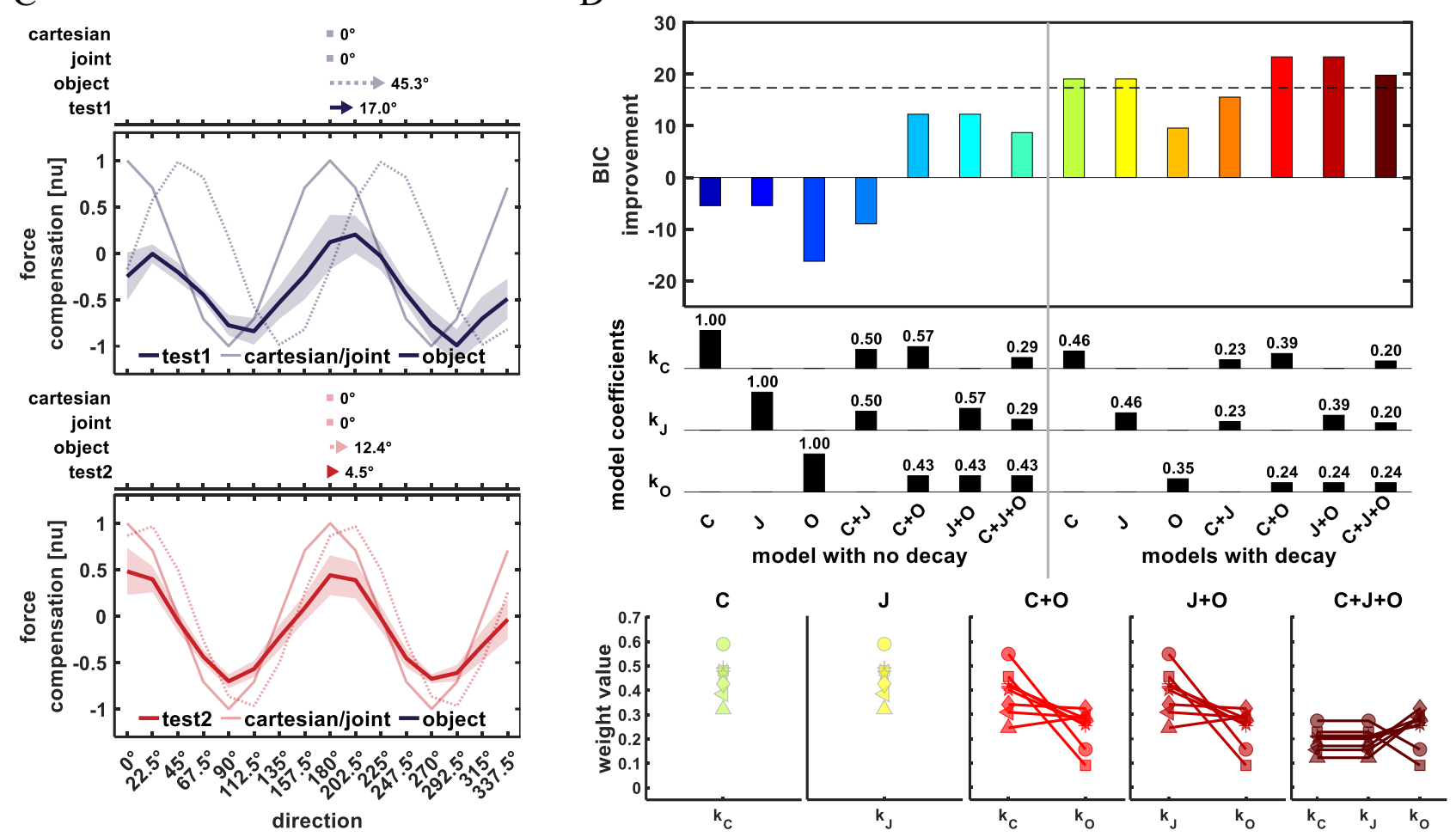

Figure 5: Results of experiment 3. All notations are similar to Figure 3.

A) Mean maximum perpendicular error (MPE).

B) Left panel, mean force compensation profiles in the training and test workspaces. Right panel, individual force compensation profiles in the training and test workspaces.

C) Upper panel, mean force compensation profile for workspace 1 and model predictions (same as Fig. 2H). Bottom panel, mean force compensation profile for workspace 2 and model predictions (same as Fig. 2I).

Arrows at the top of the panels indicate the shift of the mean force compensation profile and the models predictions in respect to the original curve describing the force field.

D) Upper panel, BIC Improvement for each of the models relative to no generalization. Dashed line shows the cutoff for models that are not considered distinguishable in terms of their performance from the best model ( $\mathrm{C}+\mathrm{O}$ or $\mathrm{J}+\mathrm{O}$ models). Middle panel, the fitted coefficients for each model. C- Cartesian, J- joint, Oobject. Bottom panels are the fitted coefficients for the best models $(\mathrm{C}, \mathrm{J}, \mathrm{C}+\mathrm{O}, \mathrm{J}+\mathrm{O}$, and $\mathrm{C}+\mathrm{J}+\mathrm{O})$ when fitting the models to individual participant data. 
432 In experiment 4, we found similar adaptation to the scaled force field as evident by the reduction

433 in MPE (Figure 6A). In this experiment, we set the location of the training and test workspaces in

434 such way that the hand orientation did not change across workspaces. In this case, both the

435 Cartesian and object-based representations predict that the curves extracted from movements in

436 the test workspaces will resemble the one extracted from the training workspace. Contrary to these

437 predictions, the joint-based representation predicts a leftward shift for the force compensation

438 curve extracted from test workspaces 1 and a rightward shift for the curve extracted from test

439 workspaces 2. For the curve calculated based on movements in the training workspace (Figure 6B,

440 grey curve) we did not find significant shift in respect to the original curve describing the force

441 field $\left(-0.03 \pm 0.07^{\circ}\right.$, mean \pm STD). For test workspace 1 , we found a small leftward shift of $-3.7 \pm 3.1^{\circ}$

442 (Figures 6B, 6C blue curve) and for test workspace 2 we found a rightward shift of $6.8 \pm 1.8^{\circ}$

443 (Figures 6B, 6C red curve). These observations were confirmed using a repeated measures

444 ANOVA that showed a statistical significant effect of the workspace condition (Greenhouse-

445 Geisser correction $\mathrm{F}_{1.11,7.83}=41.6, \mathrm{p}<0.001$ ). Post-hoc analysis showed that the training curve was

446 not statistically significantly different from the force field curve $\left(\mathrm{t}_{7}=1, p=0.35\right)$. For both test

447 workspace 1 and 2, we found statistically significant difference between the force compensation

448 curve and both the force field curve (both $\mathrm{t}_{7}=3.36, \mathrm{p}<0.05$ ) and the training workspace force

449 compensation curve (both $\mathrm{t}_{7}=3.31, \mathrm{p}<0.05$ ). We also found significant difference between the force

450 compensation curves of workspace 1 and $2\left(\mathrm{t}_{7}=6.6, \mathrm{p}=0.001\right)$.

452 Using the BIC analysis (Figure 6D), we found that the best models to explain the results were

453 mixture models combining the Cartesian or object-based representation with the joint-based

454 representation $(\mathrm{C}+\mathrm{J}$ or $\mathrm{J}+\mathrm{O})$. The optimal weights of these models were 0.31 for the contribution

455 of the joint-based representation and 0.35 for the contribution of the Cartesian or object-based

456 representations. Another model that can explain the data was a mixture of all three representations

$457(\mathrm{C}+\mathrm{J}+\mathrm{O})$. This last mixture model had same weight value $(0.31)$ for the joint-based representation

458 as in the $\mathrm{C}+\mathrm{J}$ or $\mathrm{J}+\mathrm{O}$ models and an equally divided weight for the Cartesian and object-based

459 representation $(0.175)$ since these two representations predicted similar curves for this experiment. 


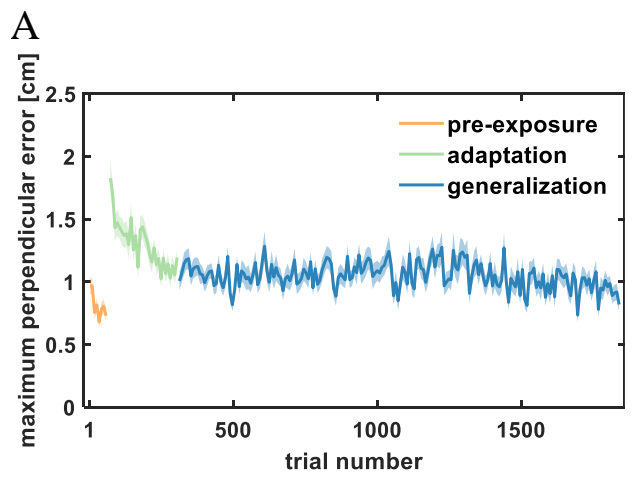

B

C
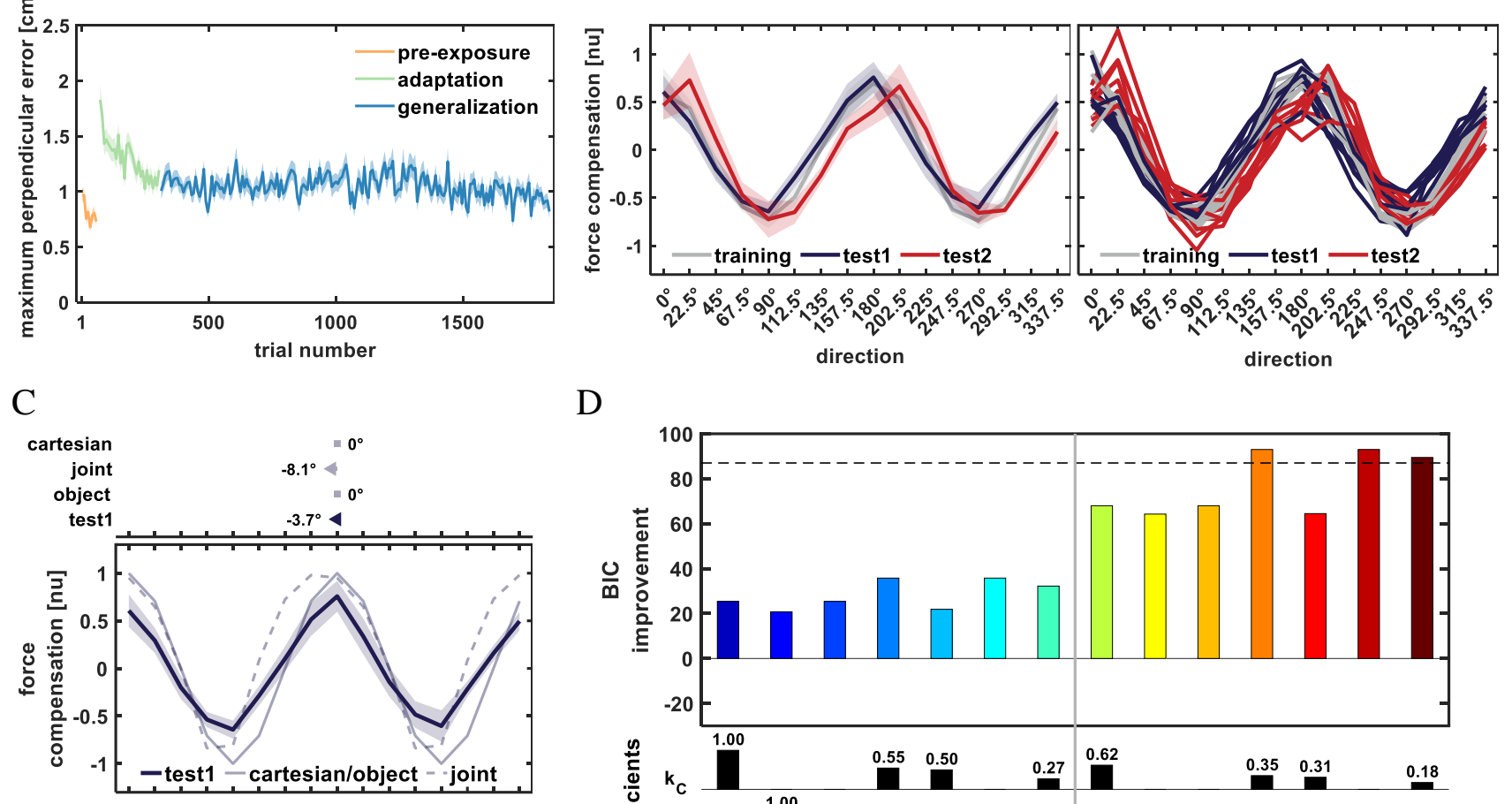

$\mathrm{D}$

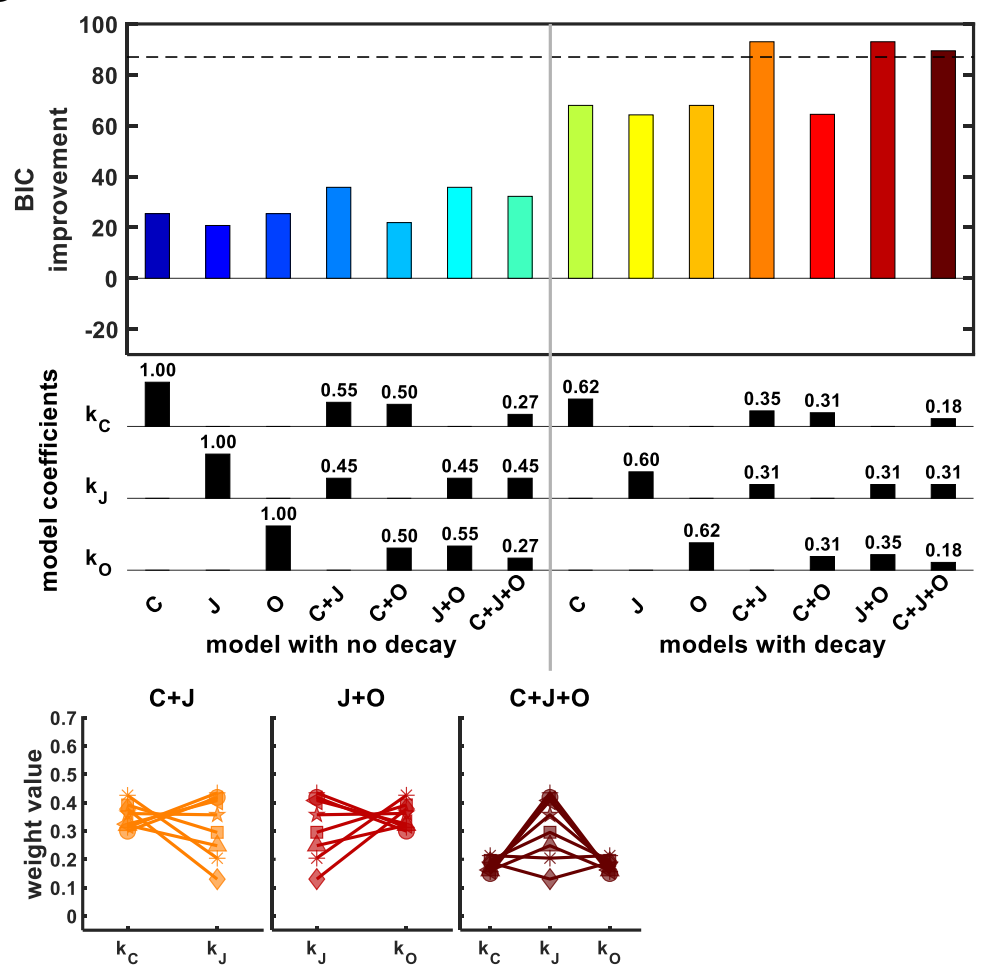

Figure 6: Results of experiment 4. All notations are similar to Figure 3.

A) Mean maximum perpendicular error (MPE).

B) Left panel, mean force compensation profiles in the training and test workspaces. Right panel, individual force compensation profiles in the training and test workspaces.

C) Upper panel, mean force compensation profile for workspace 1 and model predictions (same as Fig. 2K). Bottom panel, mean force compensation profile for workspace 2 and model predictions (same as Fig. 2L).

Arrows at the top of the panels indicate the shift of the mean force compensation profile and the models predictions in respect to the original curve describing the force field.

D) Upper panel, BIC Improvement for each of the models relative to no generalization. Dashed line shows the cutoff for models that are not considered distinguishable in terms of their performance from the best model ( $\mathrm{C}+\mathrm{J}$ or $\mathrm{J}+\mathrm{O}$ models). Middle panel, the fitted coefficients for each model. C- Cartesian, J- joint, Oobject. Bottom panels are the fitted coefficients for the best models $(\mathrm{C}+\mathrm{J}, \mathrm{J}+\mathrm{O}$, and $\mathrm{C}+\mathrm{J}+\mathrm{O})$ when fitting the models to individual participant data. 
The mixed model of Cartesian, joint- and object-based representation was the only model that explained the data across all four experiments. To further analyze the coordinate representation across all experiments, we fitted the models to the data of the four experiments combined. We

464 found that the best model was a mixed model of Cartesian, joint- and object-based representation

465 scaled by the decay factor (Figure 7). The weights for the Cartesian, joint- and object-based

representation were $0.11,0.267$ and 0.271 respectively. Moreover, only this model was selected

467 as one of the best-fitting models across all four experiments. This suggests that representation of 468 ambiguous external dynamics is formed by a mixture of Cartesian, joint- and object-based coordinate systems.

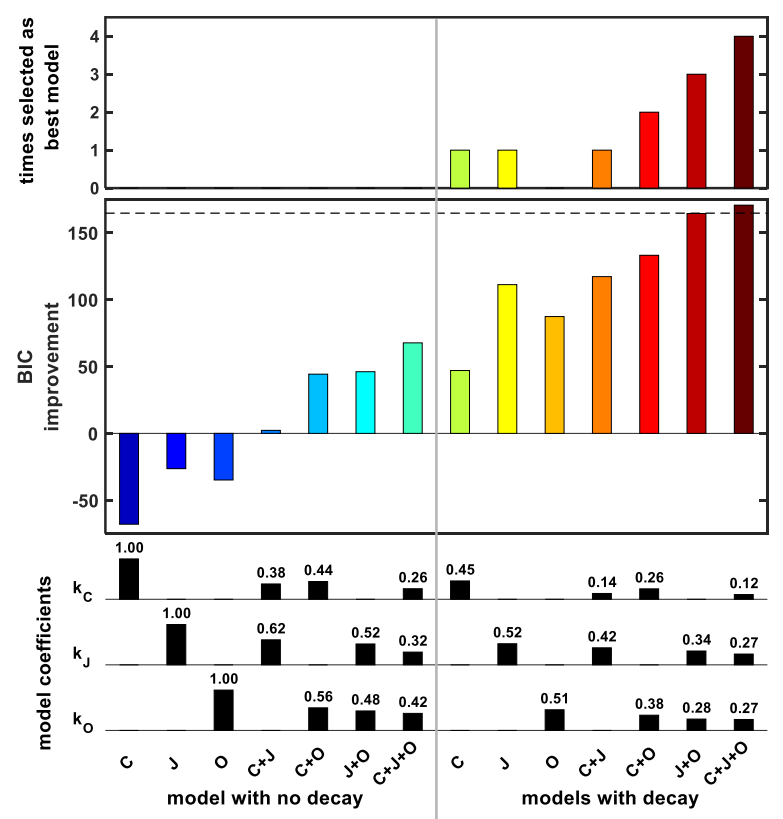

Figure 7: Upper panel shows the number of experiments that each model was able to explain when fitted to the data of each of the four experiments. Only one model $(\mathrm{C}+\mathrm{J}+\mathrm{O})$ was selected for all four experiments. Middle panel is BIC improvement for each of the models fitted to all four experiments relative to no generalization. Dashed line shows the cutoff for models that are not considered distinguishable in terms of their performance from the best model $(\mathrm{C}+\mathrm{J}+\mathrm{O}$ model $)$. All other models were below this line. Bottom panel, the fitted coefficients for each model. C- Cartesian, J- joint, Oobject. 


\section{Discussion}

472 In this study, we examined which coordinate frame the motor system uses during adaptation to 473 novel dynamics. To do so, we analyzed the pattern of generalized forces outside the training 474 workspace where participants adapted to a scaled curl force field. The trigonometric scaling of the 475 force field allowed to easily distinguish which of the three tested representations, i.e. Cartesian, 476 joint-, and object- based representations, was used to represent the external forces. We found that 477 the generalized forces resemble the prediction of a mixture model combining Cartesian, joint- and 478 object-based representations. Importantly, we show that a single reference frame could not explain 479 the generalized forces while a mixture of the models incorporating the object-based representation 480 with increased weight could explain the results. This is opposed to previous findings which 481 considered the three coordinate frames and different combinations of them but could not conclude 482 that the predictions of mixture models are superior to those of single coordinate frame based 483 models (Berniker et al., 2014).

484 The trigonometric scaling of the curl force field allowed us to distinguish between the Cartesian, 485 joint-, or object-based representations. The perpendicular forces applied on the hand during 486 movement could have different amplitude as well as different direction, i.e. clockwise or counter 487 clockwise direction, as a function of movement direction. Since the forces were perpendicular to 488 movement direction we avoided the issue of assistive or resistive forces during motion while being 489 able to capture the generalized forces using a force channel (Berniker et al., 2014). Along with 490 eliminating factors that might distort the true nature of generalized forces, the trigonometric pattern 491 of forces allowed to test models predictions by examining the phase change between adapted force 492 profile and the generalized force profile. Thus, for each experiment we choose the training and 493 testing workspaces locations so to maximize the phase difference between models predictions.

494 Initially, we tested generalization where both the posture of the arm and the orientation of the hand 495 changed between training and test locations. In such case, both the joint- and object-based 496 representations predicted a shift of the generalized forces pattern compared with the adapted force 497 pattern while the Cartesian based representation did not predict any phase shift. The shifts of the 498 curves for both test workspaces for experiment 1 and 2, as exhibited by the participants, suggest 499 that the object-based representation was slightly more dominant in representing the external forces. 
500 This result support the idea that the motor system uses this type of coordinate system as suggested

501 by previous studies (Ahmed et al., 2008; Yeo et al., 2015; Franklin et al., 2016).

502 The idea of the object-based representation suggests that the motor system relates the external

503 forces to a hand-held object, which may be a real object or not, and thus these forces should rotate

504 as the object rotates. Indeed, we observed that the generalized forces in experiment 3 rotated with

505 the rotation of the hand-held object. We observed that the expected forces were a result of

506 combining the object-based representation with either the Cartesian or joint-based representation.

507 This is in-line with previous studies which suggested that during object manipulation the motor

508 system relies on such combination of reference frames (Ahmed et al., 2008). However, other

509 studies suggested that dynamics during object manipulation are represented in joint-based

510 coordinate frame (Mah and Mussa-Ivaldi, 2003; Bays and Wolpert, 2006). As suggested by Ahmed

511 et al. (2008), a possible explanation for discrepancy might be that the motor system can gradually

512 switch between coordinate frames and this might be affected by the visual information about the

513 object or familiarity with it. The results reported here fit with this explanation since we provided

514 full visual feedback about the pole, which might trigger such transitions between coordinate

515 frames. Thus, we suggest that the greater weight of the Cartesian or joint- based representation

516 compared with the weight for the object-based representation (0.39 vs. 0.24$)$ to the overall

517 generalization forces might be a result of an incomplete transition process. Extensive training over

518 days may change this ratio in favor of the object-based representation and remains an open

519 question for future studies.

520 Another way to test the contribution of the object-based representation for dynamics representation

521 is by examining force generalization with the same hand orientation. In such case, changing joints

522 angles, and thus the arm configuration, will not change the generalized forces pattern compared

523 with the learned forces pattern. Indeed, we found small phase deviations of the force compensation

524 curves between the training and test workspaces despite the spatial difference between them. The

525 predictions of the joint-based representation were consistent with the small phase deviations we

526 found in shift direction but not in the shift magnitude. This was also confirmed by the fitting of

527 the mixture models that had increased weight for the Cartesian or object-based representation

528 compared with weight for the joint-based representation. Thus, these results suggest that the 
relative contribution of the joint-based representation is slightly lower than the contribution of the

530 object-based representation.

531 Whether there is evidence for neural encoding of an object-based representation is still an open 532 question. Given that this representation was not considered in early studies, the interpretation of 533 neural activity mostly focused on Cartesian and joint-based representations. For example, there is 534 evidence that during isometric force generation task the activity on the primary motor cortex (M1) 535 is represented in intrinsic, joint-based coordinate frame (Sergio and Kalaska, 2003). This was also 536 evident examining generalization of novel dynamics for children with autism spectrum disorder 537 (Haswell et al., 2009). However, since most efforts understating the neural basis of coordinate 538 representation focused on examining movement planning and execution, the neural representation 539 of forces is yet to be established.

540 We expect that these representations might be stored in areas related to forward and inverse 541 models. During motor adaptation, the sensorimotor system might change both the controller 542 (inverse model) and the predictor (forward model) to modify the control so as to overcome changes 543 in the environment (Flanagan et al., 2003; Franklin et al., 2008). The cerebellum has been proposed 544 to host both types of internal models (Wolpert and Kawato, 1998; Smith and Shadmehr, 2005), 545 however, other areas might also be involved during adaption to altered environment dynamics. For 546 example, changes in premotor cortex (PMd) activity were also evident during force field 547 adaptation (Perich et al., 2018). Generalization of the learned dynamics to different locations in 548 space might require from both the inverse and forward model to use dynamics transformation such 549 as the ones considered in this study. Thus, we speculate that such transformation might be evident 550 in the neural activity of these areas during adaptation to novel dynamics.

551 The results we present here support the idea that the motor system can use multiple representations 552 during adaptation to novel dynamics. However, based on these results we cannot determine the 553 trigger for favoring one coordinate frame over another. Since the force field is ambiguous (i.e. the 554 force field appeared in a small workspace with no constraints on which coordinate frame to use 555 for representation of the dynamics) it can be represented in any of the suggested coordinate frames 556 including a mixture of them. Using unambiguous force fields that extend over larger area of the 557 workspace according to one coordinate frame (Franklin et al., 2016) might result in using a single 558 coordinate frame for dynamics representation. 
559 Force generalization has been an important way to test the coordinate system the motor system 560 uses to represent information (Franklin and Wolpert, 2011). The initial idea that the motor system 561 uses intrinsic, joint-based representation (Shadmehr and Mussa-Ivaldi, 1994) is more questionable 562 nowadays as more evidence accumulated showing that the motor system can use and switch 563 between other coordinates systems (Ahmed et al., 2008; Berniker et al., 2014; Saha et al., 2015). 564 We show additional evidence that the motor system uses a mixture of models (Brayanov et al., 565 2012; Berniker et al., 2014) with slightly increased weight for the object-based representation. 566 Using a force field which overcomes some of the weaknesses other force fields have, we were able 567 to support this fairly new idea of mixture models which include an object-based representation. 568 This type of force field can be used for other types of generalization tests, such as inter-limb 569 transfer (Criscimagna-Hemminger et al., 2003) or characterizing brain related disorders (Haswell 570 et al., 2009), to reveal more characteristics of the information representation. 


\section{References}

572

573

574

575

576

577

578

579

580

581

582

583

584

585

586

587

588

589

590

591

592

593

594

595

596

597

598

599

600

601

602

603

604

605

606

607

608

609

610

611

612

613

614

Ahmed AA, Wolpert DM, Flanagan JR (2008) Flexible Representations of Dynamics Are Used in Object Manipulation. Current Biology 18:763-768.

Bays PM, Wolpert DM (2006) Actions and Consequences in Bimanual Interaction Are Represented in Different Coordinate Systems. The Journal of Neuroscience 26:71217126.

Berniker M, Franklin DW, Flanagan JR, Wolpert DM, Kording K (2014) Motor learning of novel dynamics is not represented in a single global coordinate system: evaluation of mixed coordinate representations and local learning. Journal of Neurophysiology 111:1165-1182.

Brayanov JB, Press DZ, Smith MA (2012) Motor Memory Is Encoded as a Gain-Field Combination of Intrinsic and Extrinsic Action Representations. The Journal of Neuroscience 32:14951-14965.

Criscimagna-Hemminger SE, Donchin O, Gazzaniga MS, Shadmehr R (2003) Learned Dynamics of Reaching Movements Generalize From Dominant to Nondominant Arm. Journal of Neurophysiology 89:168-176.

Flanagan JR, Vetter P, Johansson RS, Wolpert DM (2003) Prediction Precedes Control in Motor Learning. Current Biology 13:146-150.

Franklin David W, Wolpert Daniel M (2011) Computational Mechanisms of Sensorimotor Control. Neuron 72:425-442.

Franklin DW, Batchelor AV, Wolpert DM (2016) The Sensorimotor System Can Sculpt Behaviorally Relevant Representations for Motor Learning. eneuro 3:ENEURO.00700016.2016.

Franklin DW, Burdet E, Peng Tee K, Osu R, Chew C-M, Milner TE, Kawato M (2008) CNS Learns Stable, Accurate, and Efficient Movements Using a Simple Algorithm. The Journal of Neuroscience 28:11165-11173.

Gandolfo F, Mussa-Ivaldi FA, Bizzi E (1996) Motor learning by field approximation. Proceedings of the National Academy of Sciences 93:3843-3846.

Haswell CC, Izawa J, Dowell LR, Mostofsky SH, Shadmehr R (2009) Representation of internal models of action in the autistic brain. Nature neuroscience 12:970-972.

Howard IS, Ingram JN, Wolpert DM (2009) A modular planar robotic manipulandum with endpoint torque control. Journal of Neuroscience Methods 181:199-211.

Mah CD, Mussa-Ivaldi FA (2003) Generalization of Object Manipulation Skills Learned without Limb Motion. The Journal of Neuroscience 23:4821-4825.

Malfait N, Shiller DM, Ostry DJ (2002) Transfer of Motor Learning across Arm Configurations. The Journal of Neuroscience 22:9656-9660.

Milner TE, Franklin DW (2005) Impedance control and internal model use during the initial stage of adaptation to novel dynamics in humans. The Journal of Physiology 567:651664.

Oldfield RC (1971) The assessment and analysis of handedness: The Edinburgh inventory. Neuropsychologia 9:97-113.

Parmar PN, Huang FC, Patton JL (2011) Simultaneous coordinate representations are influenced by visual feedback in a motor learning task. In: 2011 Annual International Conference of the IEEE Engineering in Medicine and Biology Society, pp 6762-6768. 
615 Perich MG, Gallego JA, Miller LE (2018) A Neural Population Mechanism for Rapid Learning.

$616 \quad$ Neuron 100:964-976.e967.

617 Saha DJ, Hu X, Perreault E, Murray W, Mussa-Ivaldi FA (2015) The coordinate system for force control. Experimental Brain Research 233:899-908.

620 of Neurophysiology 86:971-985.

621 Sergio LE, Kalaska JF (2003) Systematic Changes in Motor Cortex Cell Activity With Arm Posture During Directional Isometric Force Generation. Journal of Neurophysiology 89:212-228.

Shadmehr R, Mussa-Ivaldi F (1994) Adaptive representation of dynamics during learning of a motor task. The Journal of Neuroscience 14:3208-3224.

Smith MA, Shadmehr R (2005) Intact Ability to Learn Internal Models of Arm Dynamics in Huntington's Disease But Not Cerebellar Degeneration. Journal of Neurophysiology 93:2809-2821.

Smith MA, Ghazizadeh A, Shadmehr R (2006) Interacting Adaptive Processes with Different Timescales Underlie Short-Term Motor Learning. PLOS Biology 4:e179.

Wolpert DM, Kawato M (1998) Multiple paired forward and inverse models for motor control. Neural Networks 11:1317-1329.

Yeo S-H, Wolpert DM, Franklin DW (2015) Coordinate Representations for Interference Reduction in Motor Learning. PLoS One 10:e0129388-e0129388. 